JPE 11-4-8

\title{
A Novel Seamless Direct Torque Control for Electric Drive Vehicles
}

\author{
Ahmad Ghaderi ${ }^{\dagger *}$, Takaji Umeno*, Yasushi Amano*, and Sugai Masaru* \\ ${ }^{\dagger}$ Research \& Development Dep. V, Shiga Technical Center, NIDEC Corporation, Shiga, Japan \\ ${ }^{*}$ HV Electric System Laboratory, Toyota Central R\&D Labs., Inc., Aichi, Japan
}

\begin{abstract}
Electric drive vehicles (EDV) have received much attention recently because of their environmental and energy benefits. In an EDV, the motor drive system directly influences the performance of the propulsion system. However, the available DC voltage is limited, which limits the maximum speed of the motors. At high speeds, the inverter voltage increases if the square wave (SW) voltage (six-step operation) is used. Although conventional direct torque control (DTC) has several advantages, it cannot work in the six-step mode required in high-speed applications. In this paper, a single-mode seamless DTC for AC motors is proposed. In this scheme, the trajectory of the reference flux changes continuously between circular and hexagonal paths. Therefore, the armature voltage changes smoothly from a high-frequency switching pattern to a square wave pattern without torque discontinuity. In addition, because multi-mode controllers are not used, implementation is more straightforward. Simulation results show the voltage pattern changes smoothly when the motor speed changes, and consequently, torque control without torque discontinuity is possible in the field weakening area even with a six-step voltage pattern.
\end{abstract}

Key Words: Direct Torque Control, Motor, Overmodulation, Seamless Control, Six-step

\section{NOMENCLATURE}

$\begin{array}{lll}\omega \mathrm{r} & : & \text { Motor Speed } \\ \omega \mathrm{r}^{*} & : & \text { Reference Speed } \\ \mathrm{Te} & : & \text { Reference torque } \\ \mathrm{Te} & : & \text { Motor torque } \\ \Psi^{*} & : & \text { Reference flux } \\ \Psi^{*} \mathrm{new} & : & \text { New reference flux } \\ \Psi^{*} \mathrm{~m} & : & \text { Minimum of the reference flux } \\ \Psi^{*} \mathrm{av} & : & \text { Average of the reference flux } \\ \mathrm{Vdc} & : & \text { DC voltage } \\ \Delta \mathrm{Te} & : & \text { Torque error } \\ \Delta \Psi & : & \text { Flux error } \\ \Delta \mathrm{Te} * & : & \text { Torque hystersis } \\ \mathrm{dte} & : & \text { Torque signal } \\ \mathrm{d} \Psi & : & \text { Flux signal } \\ \operatorname{VaN}(\theta): & \text { Phase voltage } \\ \rho \mathrm{s} & : & \text { Phase angle } \\ \theta \mathrm{r} & : & \text { Rotor angle } \\ \omega \mathrm{c} & : & \text { Crossover speed } \\ \Psi \max & : & \text { Maximum of the reference flux } \\ \Psi \min * & : & \text { Minimum of the reference flux } \\ \operatorname{Delta}(\delta): & \text { Angle of non- switching area } \\ \alpha & : & \text { Angle between phase angle and center of } \\ & & \text { delta }\end{array}$

Manuscript received Dec. 28, 2010; revised May 18, 2011

Recommended for publication by Guest Associate Editor Chris Edrington.

$\dagger$ Corresponding Author: ahmad.ghaderi@jp.nidec.com

Tel: +81-749-42-6038, Fax: +81-749-42-6182, NIDEC Corp

* HV Electric System Laboratory, Toyota Central R\&D Labs., Inc., Japan

\section{INTRODUCTION}

In recent years, electric drive vehicles (EDVs) have received much attention because they can reduce environmental and energy problems. In all types of EDVs, including electric vehicles, electric hybrid vehicles, and fuel cell vehicles, the performance of the electric motor and its drive system affect the vehicle handling, the ride quality, and the maximum travel range. Therefore, a comprehensive design of propulsion motors and their controller systems is essential [1]-[4].

The conventional structure of motor drives for electric vehicles is shown in Fig. 1. As shown in this figure, the available DC link voltage of the inverter $(\mathrm{Vdc})$ depends on the battery level and the booster converter. When the battery voltage is limited, the voltage of the booster converter is also limited because of circuit isolation and safety issues. Consequently, the DC link voltage of the inverter is limited, which in turn limits the inverter output. Because the backEMF of the motor armature increases at higher speeds due to the limited voltage, the armature current and consequently the motor torque can be limited. Thus, for a pre-fixed Vdc, the motor speed and consequently the vehicle speed are limited [5].

If the inverter switching pattern changes from a sine wave to a square wave (SW), the inverter voltage and consequently the maximum speed of the motor increase. The voltage of a square wave is approximately $27 \%$ higher than that of a sinusoidal wave with equal peaks. In addition, with a square pattern, the switching loss decreases. Because the SW pattern includes the harmonics of the fundamental frequency, this pattern should 
TABLE I

MOTOR SPECIFICATIONS THE CORRESPONDING 3-PHASE VOLTAGE OF THE VOLTAGE VECTORS

\begin{tabular}{|c|c|c|c|c|c|}
\hline Vector & VcN & VbN & VaN & Vbc & Angle \\
\hline U0 & 0 & 0 & 0 & 0 & For $\delta=0$ \\
\hline U1 & Vdc & 0 & 0 & $-\mathrm{Vdc}$ & $-\pi / 3+\delta / 2<\alpha<\pi / 3-\delta / 2$ \\
\hline U2 & Vdc & Vdc & 0 & 0 & $\delta / 2<\alpha<2 \pi / 3-\delta / 2$ \\
\hline U3 & 0 & $\mathrm{Vdc}$ & 0 & $\mathrm{Vdc}$ & $\pi / 3+\delta / 2<\alpha<\pi-\delta / 2$ \\
\hline U4 & 0 & $\mathrm{Vdc}$ & $\mathrm{Vdc}$ & $\mathrm{Vdc}$ & $2 \pi / 3+\delta / 2<\alpha<4 \pi / 3-\delta / 2$ \\
\hline U5 & 0 & 0 & $\mathrm{Vdc}$ & 0 & $\pi+\delta / 2<\alpha<5 \pi / 3-\delta / 2$ \\
\hline U6 & $\mathrm{Vdc}$ & 0 & $\mathrm{Vdc}$ & $-\mathrm{Vdc}$ & $4 \pi / 3+\delta / 2<\alpha<2 \pi-\delta / 2$ \\
\hline U7 & $\mathrm{Vdc}$ & $\mathrm{Vdc}$ & $\mathrm{Vdc}$ & 0 & For $\delta=0$ \\
\hline
\end{tabular}

only be applied at higher speeds, at which the motor acts as a low-pass filter. At lower speeds, the sinusoidal switching pattern should be employed, as shown in Fig.2. To avoid torque discontinuity, switching between these patterns must be done seamlessly [6].

However, for traction applications, the motor drive needs a fast torque response with a straightforward mechanism. Especially for vehicles with multi-motor propulsion systems include in-wheel motors, fast torque response is very essential [7]. A comparison of various motor drives shows direct torque control (DTC) drive has several advantages and the torque response is much faster than any AC or DC drive [8]. However, with a conventional DTC, the square wave or the six-step mode required at high speeds is not possible [9]. Therefore, to achieve an accurate seamless transformation between the conventional DTC and the six-step operation, several studies are now being conducted.

In some studies, third harmonics injection with space vector modulation (SVM) [10] or pulse-width modulation (PWM) [11] have been employed. With this method, the fundamental voltage is less because the complete six-step mode cannot be achieved. In [12], space vector PWM with a pulse-dropping algorithm is proposed; however, in this method the control over modulation decreases and the motor harmonic losses grow. The DTC-SVM proposed in [13] requires complicated calculations and cannot work completely in the six-step mode [14]. In [15], a novel DTC-SVM with a fast torque response and the capability of six-step operation is proposed. However, in this scheme, as in other DTC-SVM methods, complicated calculations are essential and a fast digital processor is required [13]. In all the abovementioned methods, generating the reference voltage vector is essential. In contrast, in hysteresis based DTC, control is achieved without a reference voltage vector.

In some researches multi-mode control algorithms are used in order to achieve a smooth transition between different patterns for reference voltage [14], [16]. In [14] and [16] the control mode is changed considering a certain time interval. However it is not clear how to determine the length of time interval. On the other hand, the torque discontinuity may be appear during the switching between control modes. In order to avoid this torque fluctuation, a sophisticated mechanism is essential, which leads to system complexity.

Although hysteresis based DTC is very straightforward, no research on hysteresis based DTC with six-step capability has been reported [14].

To avoid mentioned problems, in this paper, a new hysteresis based DTC capable of six-step operation is proposed. Because the conventional DTC cannot work in six-step mode, we develop a new DTC with a modified flux trajectory. In this approach, the motor flux trajectory is transformed continuously between a circular shape and a hexagonal shape. Because the inverter voltage changes from high-frequency switching to a six-step square wave with only one control mode, seamless torque control from a constant torque area to a constant power region, and vice versa, is possible.

\section{The Proposed DTC}

To activate the target-switching pattern in the proposed method, the flux trajectory is modified. Fig. 3 shows the trajectory of the reference flux. As shown in this figure, if the angle of $\delta$ is changed between 0 to 60 degrees, the flux trajectory changes from a circular to a hexagonal shape continuously. In this figure, U1-U6 indicates the voltage vectors, which are selected based on the motor flux and the phase angle. The corresponding 3-phase voltage of the voltage vectors is shown in Table I.

The block diagram of the proposed method is shown in Fig. 4. As shown in this figure, the reference flux trajectory is not constant, unlike that in conventional hysteresis based DTC. The maximum and the minimum of the reference flux are defined considering the phase angle and $\delta$. The value of $\delta$ is calculated based on the required fundamental component. The fundamental component is defined based on the following: inverter voltage, motor torque, reference torque, motor speed, and crossover speed.

If the flux shape is changed, its average value varies. This deviation needs to be considered in the computation of the torque. Therefore, in the proposed method a torque compensator unit is added to compensate for the effect of the flux variation. In addition, the switching pattern is modified to avoid unwanted switching when the flux sector is changed.

\section{The Reference FluX Trajectory Modifier}

If the reference flux is calculated properly, the flux trajectory shifts continuously from a circular path to a hexagonal path. The relation of the reference flux and the phase angle is shown in Fig. 5. As shown in this figure, the new reference flux is a function of the phase angle and $\delta$. The new reference flux amplitude is calculated in (1)-(2) considering Fig. 3.

$$
\begin{aligned}
& \psi_{\mathrm{m}}^{*}=\psi^{*} \cos \left(\frac{\delta}{2}\right) \quad \text { for }-\frac{\delta}{2}+n \frac{\pi}{3} \leq \alpha \leq \frac{\delta}{2}+n \frac{\pi}{3} \\
& (n=1 \text { to } 6) \\
& \psi_{\mathrm{m}}^{*}=\psi^{*} \text { for other } \alpha \\
& \psi_{\text {new }}^{*}=\frac{\psi_{m}^{*}}{\cos (\alpha)}=\frac{\cos \left(\frac{\delta}{2}\right)}{\cos (\alpha)} \psi^{*} \\
& \text { for }-\frac{\delta}{2}+n \frac{\pi}{3} \leq \alpha \leq \frac{\delta}{2}+n \frac{\pi}{3} \\
& (n=1 \text { to } 6) \\
& \psi_{\mathrm{m}}^{*}=\psi^{*} \text { for other } \alpha \text {. }
\end{aligned}
$$


Inverter

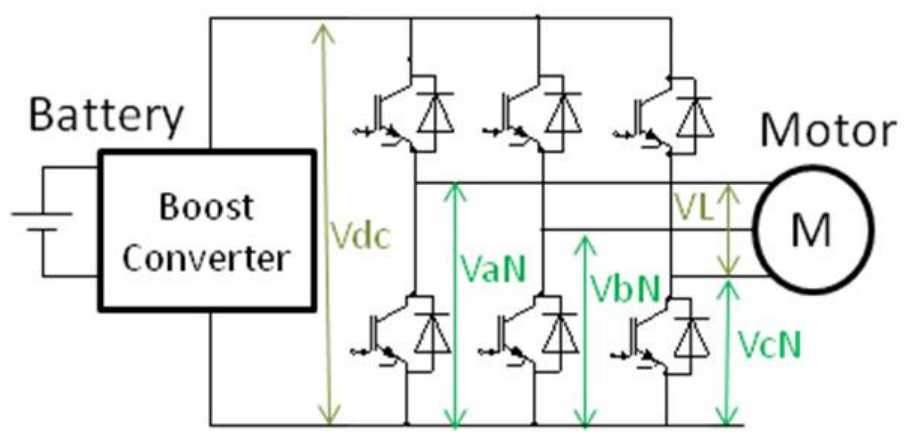

Fig. 1. Structure of an AC Motor Drive for Vehicle Applications.
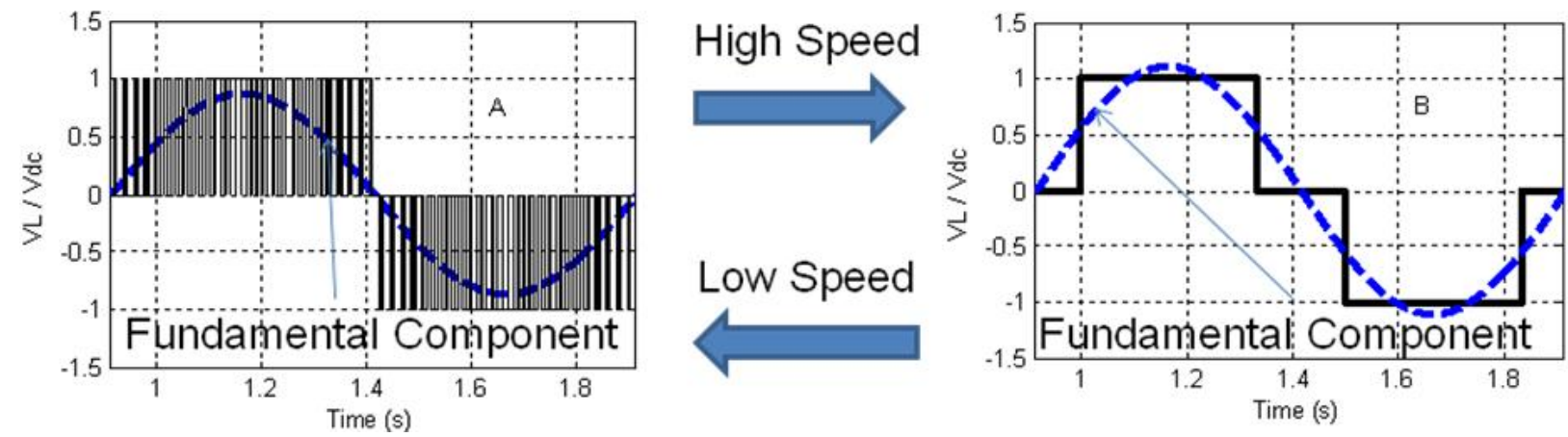

Fig. 2. Comparison between (A) the sine wave and (B) the square wave.

\section{Compensation of THE ReFEREnce Torque}

As shown in Fig. 5, when $\delta$ changes, the flux average also varies. For example, if $\delta$ increases, the flux average is reduced, which leads to torque reduction. For proper control, the effect of the variation of $\delta$ on the torque should be compensated. To calculate the flux reduction, the reference flux average is calculated by integration of the reference flux, as follows.

$$
\begin{gathered}
\psi_{\mathrm{av}}^{*}=\frac{3}{\pi} \int_{-\frac{\pi}{6}}^{+\frac{\pi}{6}} \psi_{\text {new }}^{*} d \alpha= \\
\frac{3}{\pi} \psi^{*}\left[\left(\frac{\pi}{3}-\delta\right)+\cos \left(\frac{\delta}{2}\right) \int_{-\frac{\pi}{6}}^{+\frac{\pi}{6}} \frac{d \alpha}{\cos (\alpha)}\right] \\
\psi_{\mathrm{av}}^{*}=\frac{3}{\pi} \psi^{*}\left[\left(\frac{\pi}{3}-\delta\right)+\cos \left(\frac{\delta}{2}\right) \ln \left(\frac{1+\sin \left(\frac{\delta}{2}\right)}{1-\sin \left(\frac{\delta}{2}\right)}\right)\right] .
\end{gathered}
$$

The difference between the reference flux and the average of the new reference flux is as follows.

$$
\psi_{\mathrm{av}}^{*}-\psi^{*}=\frac{3}{\pi} \psi^{*}\left[\delta-\cos \left(\frac{\delta}{2}\right) \ln \left(\frac{1+\sin \left(\frac{\delta}{2}\right)}{1-\sin \left(\frac{\delta}{2}\right)}\right)\right] .
$$

\section{Calculation of the Fundamental Component}

In DTC control based on the phase angle, two sequential voltage vectors and a zero voltage vector are applied. However, as the speed increases, the required reference voltage increases and thus the duty cycle of the zero vector decreases. At the crossover speed, only the non-zero voltage vectors are applied to the motor. In our purposed method, when the speed becomes higher than the crossover speed, the angle of $\delta$ increases from zero. In this situation, one vector in the linear part and two sequential vectors in the circular path of the flux trajectory are applied. The duty cycle of the vectors depends on the phase angle. For example, as shown in Table I, for $\pi / 3+\delta / 2<\alpha<2 \pi / 3-\delta / 2$, U2 and U3 are applied. To calculate the duty cycle of $\mathrm{U} 2$ and $\mathrm{U} 3$, the variation of the flux $(\Delta \psi)$ caused by these vectors is shown in Fig. 6. Here, D2 and D3 are the duty cycles of $\mathrm{U} 2$ and $\mathrm{U} 3$, respectively.

Because the amplitude of U2 equals the amplitude of U3, D2 and D3 are calculated as follows.

$$
\begin{aligned}
D_{2} & =\frac{\left|U_{2} T_{2}\right|}{\left|U_{2} T_{2}\right|+\left|U_{3} T_{3}\right|}=\frac{\left|\sin \left(\frac{\pi}{6}-\theta\right)\right|}{\left|\sin \left(\frac{\pi}{6}+\theta\right)\right|+\left|\sin \left(\frac{\pi}{6}-\theta\right)\right|} \\
& =\frac{1-\sqrt{3} \tan \theta}{2} \\
D_{3} & =\frac{\left|U_{3} T_{3}\right|}{\left|U_{2} T_{2}\right|+\left|U_{3} T_{3}\right|}=\frac{\left|\sin \left(\frac{\pi}{6}+\theta\right)\right|}{\left|\sin \left(\frac{\pi}{6}+\theta\right)\right|+\left|\sin \left(\frac{\pi}{6}-\theta\right)\right|} \\
& =\frac{1+\sqrt{3} \tan \theta}{2}
\end{aligned}
$$

Therefore, (8) is achieved considering Table I and (6).

$$
\begin{gathered}
V c N(\theta)=V d c D_{2}=\frac{V d c}{2}(1-\sqrt{3} \tan \theta) \\
\text { for }-\frac{\pi}{6}+\frac{\delta}{2} \leq \theta \leq \frac{\pi}{6}-\frac{\delta}{2} .
\end{gathered}
$$

Using a similar approach, $\mathrm{VcN}$ is calculated when U5 and 


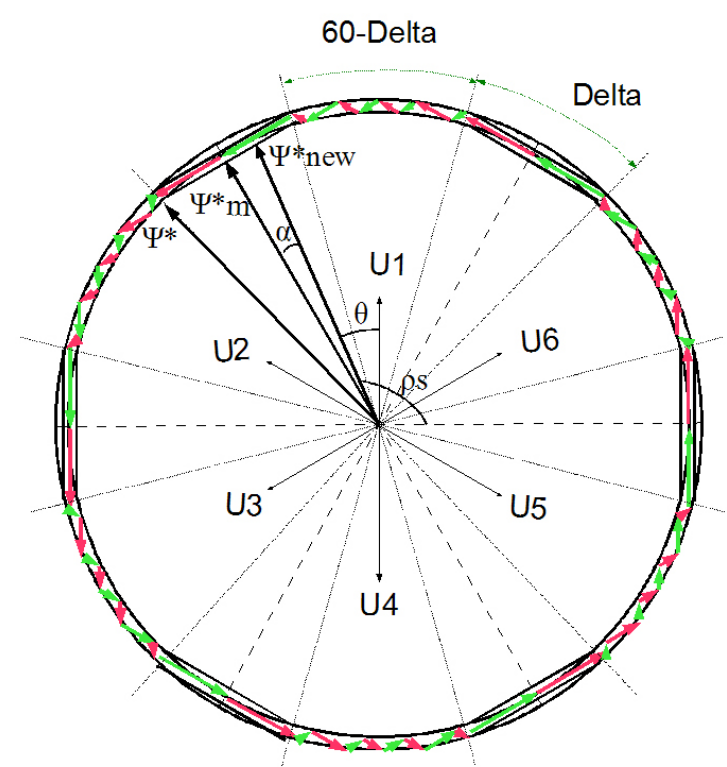

Fig. 3. The reference flux trajectory.

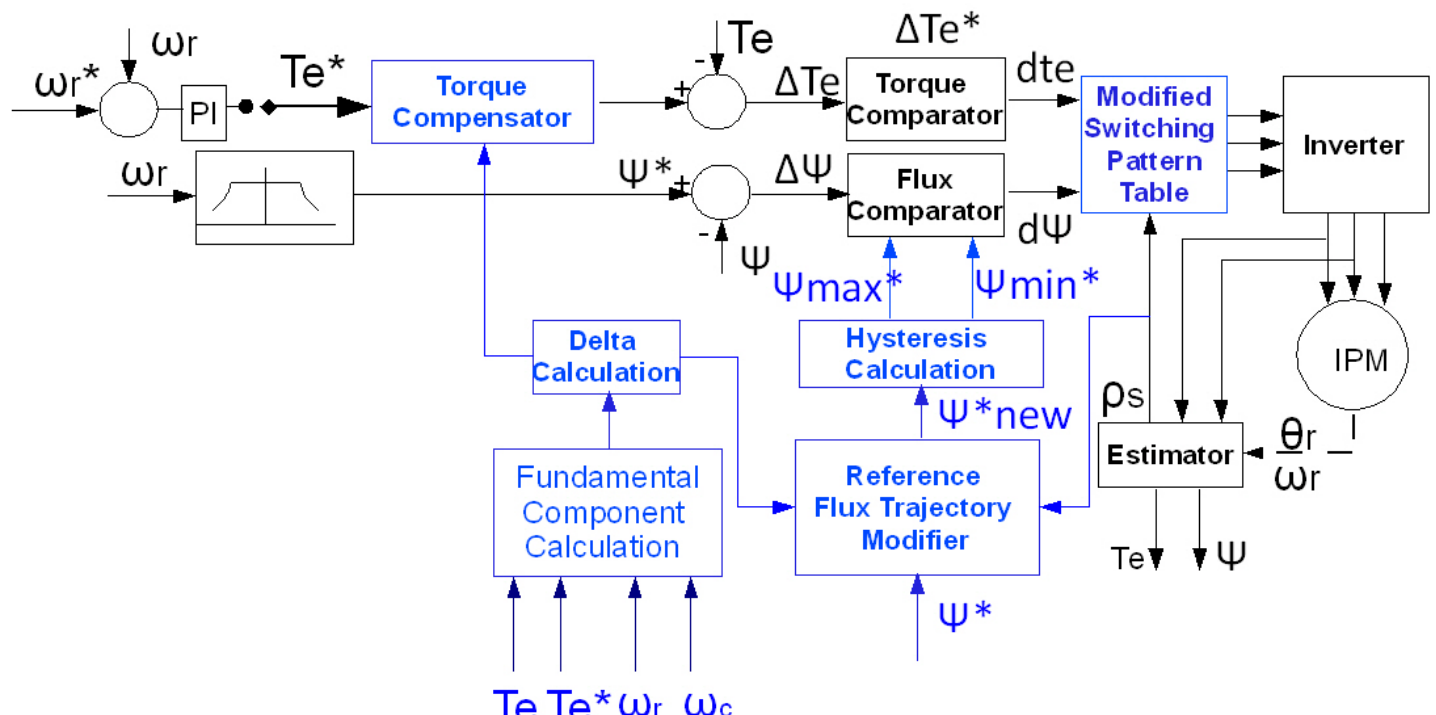

Fig. 4. Block diagram of the proposed DTC.

U6 are applied sequentially, as shown in (9).

$$
\begin{array}{r}
V c N(\theta)=\frac{V d c}{2}(1+\sqrt{3} \tan (\theta-\pi)) \\
\text { for } \frac{5 \pi}{6}+\frac{\delta}{2} \leq \theta \leq \frac{7 \pi}{6}-\frac{\delta}{2} .
\end{array}
$$

For the other angles, $\mathrm{VcN}$ is calculated in (10) and (11).

$$
\begin{gathered}
V c N(\theta)=0 \\
\text { for } \frac{\pi}{6}-\frac{\delta}{2} \leq \theta \leq \frac{\pi}{6}+\frac{\delta}{2} \\
V c N(\theta)=V d c \\
\text { for }-\frac{5 \pi}{6}-\frac{\delta}{2} \leq \theta \leq-\frac{\pi}{6}+\frac{\delta}{2} .
\end{gathered}
$$

The coefficient of the fundamental voltage (b1) is calculated by using Fourier coefficients of the voltage, as follows.

$$
b_{n}=\frac{1}{\pi} \int_{-\pi}^{\pi} V c n(\theta) \sin (n \theta) d \theta=\frac{4}{\pi} \int_{0}^{\frac{\pi}{2}} V c n(\theta) \sin (n \theta) d \theta
$$

$$
a_{n}=\frac{4}{\pi} \int_{0}^{\frac{\pi}{2}} V c n(\theta) \cos (n \theta) d \theta=0 \quad n \geq 1
$$

$$
\left|b_{1}\right|=\frac{4}{\pi} \int_{0}^{\frac{\pi}{6}-\frac{\delta}{2}} \sqrt{3} \frac{V d c}{2} \tan (\theta) \sin (\theta) d \theta+\frac{4}{\pi} \int_{\frac{\pi}{6}-\frac{\delta}{2}}^{\frac{\pi}{2}} \frac{V d c}{2} d \theta
$$




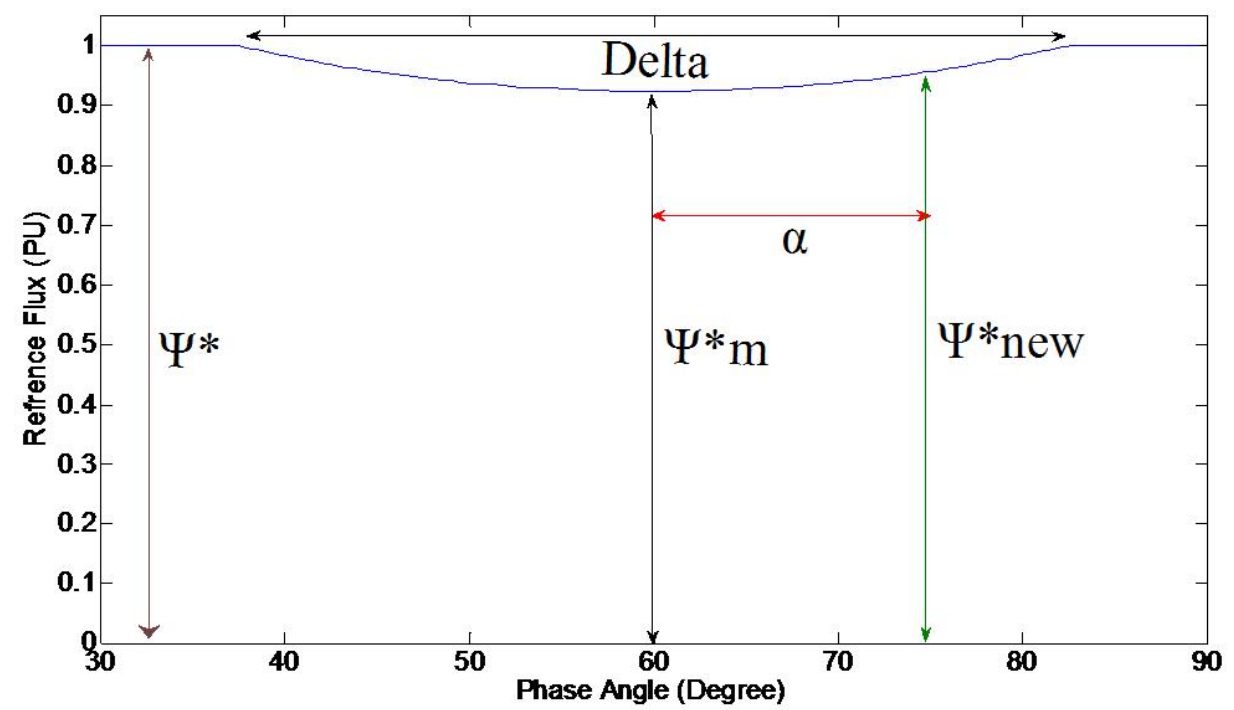

Fig. 5. The relation of the reference flux and the phase angle.

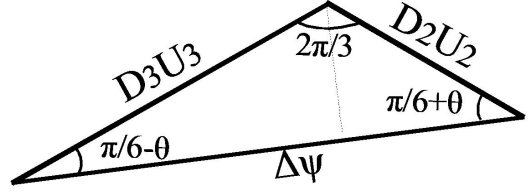

Fig. 6. The variation of the flux caused by $\mathrm{U} 2$ and $\mathrm{U} 3$ for $-\pi / 6+\delta / 2<\theta<$ $\pi / 6-\delta / 2$.
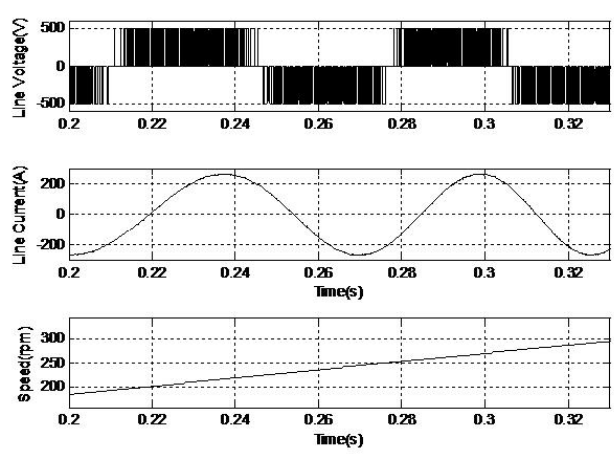

Fig. 7. Voltage, current, and speed for low-speed area
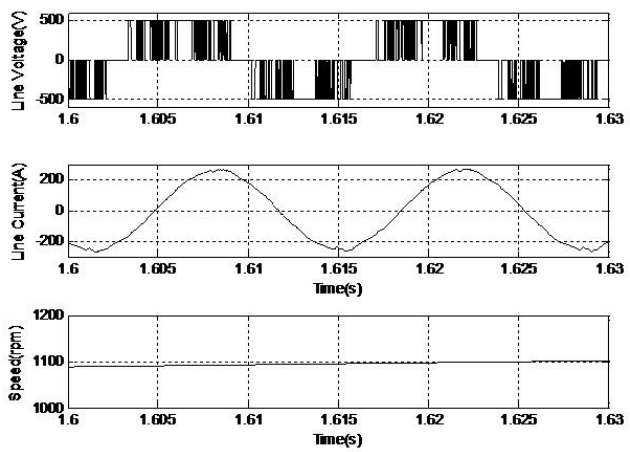

Fig. 8. Voltage, current, and speed for middle-speed area.
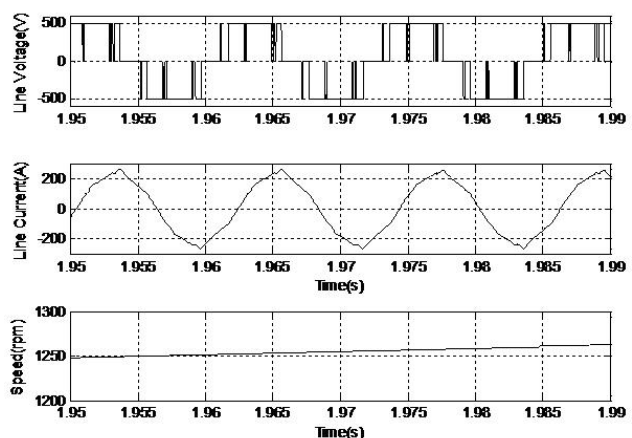

Fig. 9. Voltage, current, and speed for high-speed area.
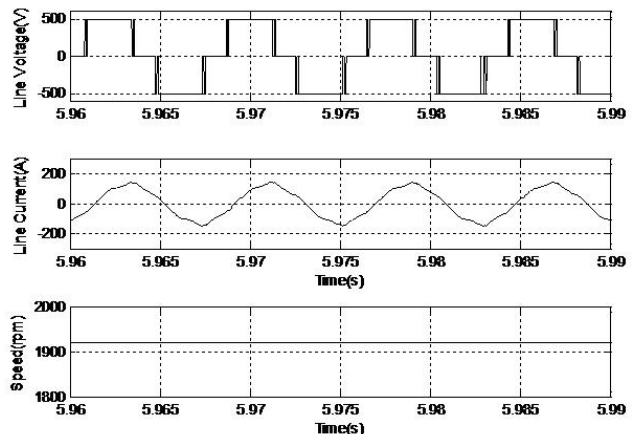

Fig. 10. Voltage, current, and speed for the maximum speed.

$$
\begin{aligned}
\left|b_{1}\right| & =\frac{2 \sqrt{3} V d c}{\pi}\left[\ln \left|\tan \left(\frac{\pi}{3}-\frac{\delta}{4}\right)\right|-\sin \left(\frac{\pi}{6}-\frac{\delta}{2}\right)\right] \\
& +\frac{2 V d c}{\pi} \cos \left(\frac{\pi}{6}-\frac{\delta}{2}\right) .
\end{aligned}
$$

Equation (15) expresses the relation between b1 and $\delta$. The $\delta$ angle can be calculated as a function of the required fundamental voltage by interpolation of the data of (15). 

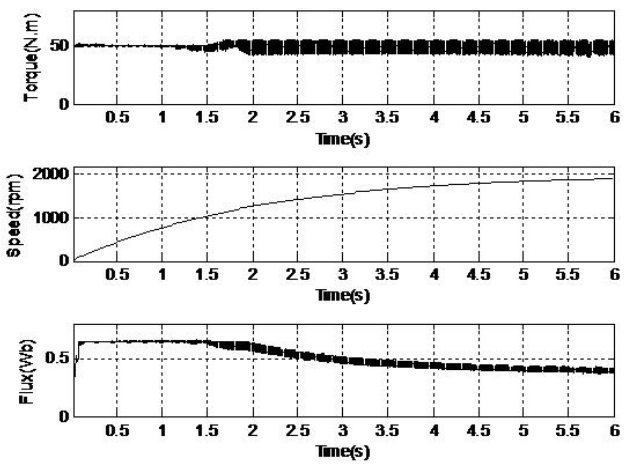

Fig. 11. Torque, speed, and flux for 0 to maximum speed.
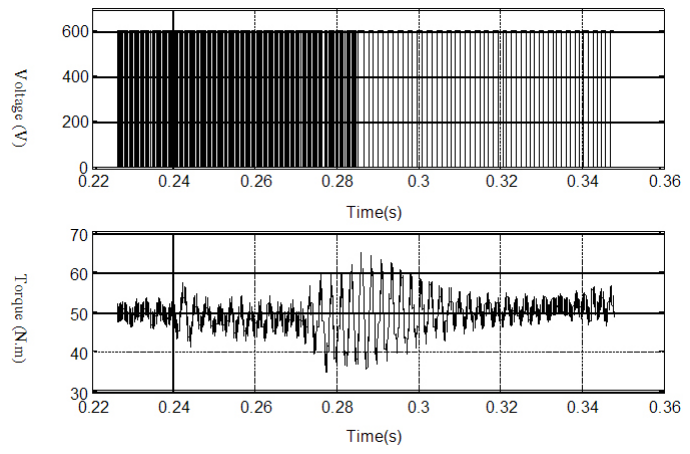

Fig. 12. Voltage and torque for a multi-mode controller.

\section{Simulation Results}

In this paper, the simulation of the proposed DTC is performed using Ansoft Simplorer with a surface permanentmagnet synchronous motor, which has the parameters shown in Table II. The simulation results are shown in Fig. 7-Fig. 11. As shown in Fig. 7-Fig. 10, at low speeds the voltage pattern is similar to a conventional DTC, but, as the speed increases, the voltage pattern changes smoothly to the square wave. Likewise, at lower speeds, the motor current is completely sinusoidal, and at higher speeds, the motor current shape slightly deviates from the sinusoidal wave because of the application of the square wave voltage.

Fig. 11 shows the torque, speed, and flux when the speed changes from zero speed to the maximum speed. These results show that torque control is possible in the field weakening area even with the six-step voltage pattern without torque discontinuity. In addition, as shown in the simulation results, there is some torque ripple at higher speeds when the square wave voltage is applied. However, the ripple does not appear in the motor speed because at higher speeds the motor acts as a low-pass filter.

The simulation results of a multi-mode controller [15], undertaken using an almost similar motor, is shown in Fig. 12. As seen in this figure, the torque discontinuity appears during control mode changing. Comparing figures 11 and 12 shows that in our proposed method, the torque discontinuity is lower than multi-mode controllers.
TABLE II

PARAMETERS OF THE SURFACE PERMANENT-MAGNET SYNCHRONOUS MOTOR

\begin{tabular}{|c|c|}
\hline Rotor Resistance & $0.0522 \mathrm{Ohm}$ \\
\hline Stator Inductance d-axis & $1.8 \mathrm{mH}$ \\
\hline Stator Inductance q-axis & $1.8 \mathrm{mH}$ \\
\hline Rotor Flux & $0.16 \mathrm{~Wb}$ \\
\hline Number of Pole Pairs & 4 \\
\hline Vdc & 500 Volt \\
\hline
\end{tabular}

\section{CONCLUSIONS}

In this paper, a novel single-mode seamless DTC for AC motors is proposed. Though seamless control is not possible by conventional DTC, our proposed method can apply torque control without torque discontinuity. In our method, the motor flux trajectory is modified to change between circular and hexagonal paths continuously. Therefore, the inverter voltage changes smoothly from high-frequency switching to a square wave with only one control mode. This algorithm is more straightforward than current technologies, which are based on multi-mode controllers. The simulation results show that at low speeds the voltage has a high-frequency switching pattern, but changes smoothly to the square wave as the speed increases. The torque control without torque discontinuity is possible in the field weakening area even with the six-step voltage pattern.

\section{REFERENCES}

[1] W. Li, J. Cao, and X. Zhang, "Electrothermal analysis of induction motor with compound cage rotor used for P," IEEE Trans. Ind. Electron., Vol. 57, No. 2, pp. 660-668, Feb. 2010.

[2] P. C. Desai, M. Krishnamurthy, N. Schofield, and A. Emadi, "Novel switched reluctance machine configuration with higher number of rotor poles than stator poles: concept to implementation," IEEE Trans. Ind. Electron., Vol. 57, No. 2, pp. 649-659, Feb. 2010.

[3] M.-J. Yang, H.-L. Jhou, B.-Y. Ma, and K.-K. Shyu, "A cost-effective method of electric brake with energy regeneration for electric vehicles," IEEE Trans. Ind. Electron., vol. 56, no. 6, pp. 2203-2212, Jun. 2009.

[4] D. Yin, S. Oh, and Y. Hori, "A novel traction control for EV based on maximum transmissible torque estimation," IEEE Trans. Ind. Electron., Vol. 56, No. 6, pp. 2086-2094, Jun. 2009.

[5] D. Casadei, G. Serra, A. Tani, and L. Zarri, "A robust method for field weakening operation of induction motor drives with maximum torque capability," IAS 2006, Oct. 2006.

[6] B. K. Bose and P. M. Szczesny, "A microcomputer-based control and simulation of an advanced IPM synchronous machine drive system for electric vehicle propulsion," IEEE Trans. Ind. Electron., Vol. 35, No. 4, pp. 547-559, Aug. 1988.

[7] K. T. Chau, C. C. Chan, L. Chunhua, "Overview of permanent-magnet brushless drives for electric and hybrid electric vehicles," IEEE Trans. Ind. Electron., Vol. 55, No. 6, pp. 2246-2257, May 2008.

[8] K.-K. Shyu, J.-K. Lin, V.-T. Pham, M.-J. Yang, and T.-W. Wang, "Global minimum torque ripple design for direct torque control of induction motor drives," IEEE Trans. Ind. Electron., Vol. 57, No. 10, pp. 31483156, Dec. 2010.

[9] A. Ghaderi, T. Umeno, Y. Amano, and M. Sugai, "A new direct torque control for AC motors with over modulation ability," IEEE-IEMDC, May 2011.

[10] D. Casadei, G. Serra, A. Tani, and L. Zarri, "A robust method for field weakening operation of induction motor drives with maximum torque capability," IEEE-IAS 2006, pp. 111-117, Oct. 2006.

[11] Z. Peroutka and K. Zeman, "Robust field weakening algorithm for vector-controlled induction machine traction drives," IECON 2006, pp. 3987-3992, Nov. 2006.

[12] H. J. Park and M. J. Youn, "A new time-domain discontinuous spacevector PWM technique in overmodulation region," IEEE Trans. Ind. Electron., Vol. 50, No. 2, pp. 349- 355, Apr. 2003. 
[13] T. G. Habetler, F. Profumo, M. Pastorelli, and L. M. Tolbert, "Direct torque control of induction machines using space vector modulation," IEEE Trans. Ind. Appl., Vol. 28, No. 5, pp. 1045-1053, Sep./Oct. 1992.

[14] A. Jidin, N. Idris, A. Yatim, and M. Elbuluk, "A novel overmodulation and field weakening strategy for direct torque control of induction machines,' IEEE IAS '08, pp. 1-8, Oct. 2008.

[15] A. Tripathi, A. M. Khambadkone, and S. K. Panda, "Dynamic control of torque in overmodulation and in the field weakening region," IEEE Trans. Power Electron., Vol. 21, No. 4, pp. 1091-1098, Jul. 2006.

[16] H. Nakai, H. Ohtani, E. Satoh, and Y. Inaguma, "Development and testing of the torque control for the permanent-magnet synchronous motor," IEEE Trans. on Ind. Electron., Vol. 52, No. 3, pp. 800-806, Jun. 2005

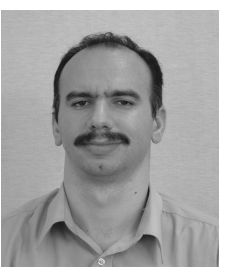

Ahmad Ghaderi received the B.S., M.S., and $\mathrm{PhD}$ in Electrical Engineering from Shahed University, Iran, Isfahan University of technology, Iran, and Kyushu Institute of Technology, Japan, in 1999, 2002, and 2007 respectively. From 2002 to 2003 he was with National Iranian Oil Refining and Distribution Company. He was a research assistance at Robotics Research Institute, Japan, from 2004 to 2006, and a researcher in Kyushu Institute of Technology, Japan, from 2007 to 2008. He worked at HV Electric System Lab., Toyota Central R\&D Labs, Inc., Japan, from 2008 to 2011. And currently he works at Shiga Technical Center, NIDEC Corporation, Japan. His research interests include power electronics, motor control, and electric drive vehicle propulsion systems.

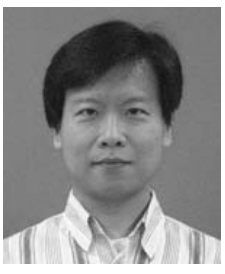

Takaji Umeno was born in Fukui Prefecture, Japan, in 1963. He received B.E and M.E. degrees in computer engineering from Toyohashi University of Technology, Toyohashi, Japan, in 1986 and 1988, respectively, and the $\mathrm{Ph} . \mathrm{D}$. degree in electrical engineering from the University of Tokyo, Tokyo, Japan, in 1991. Since 1991, he has been with Toyota Central R\&D Labs., INC. His main research interest involves power electronics and control of hybrid vehicles and electric vehicles. He is a member of the Institute of Electrical Engineers Japan (IEEJ), the Society of Instrument and Control Engineers (SICE), thenSociety of Automotive Engineers of Japan (JSAE) and IEEE.

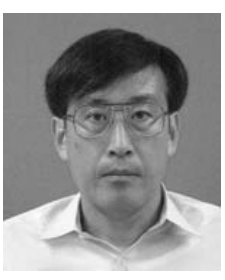

Yasushi Amano was born in Aichi Prefecture, Japan, in 1957. He received the Eng.D. degree in mechanical engineering from Sophia University, Tokyo, Japan, in 1985. Since 1985, he has been with Toyota Central R\&D Labs., INC. His current research interests are hybrid vehicle control and energy management. Dr. Amano is a member of the Society of Instrument and Control Engineers, the Japan Society of Mechanical Engineers and Society of Automotive Engineers of Japan.

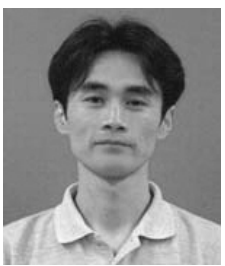

Sugai Masaru was born in Shiga Prefecture, Japan, in 1967. He received B.E and M.E. degrees in electrical engineering from the University of Tokyo, Tokyo, Japan, in 1991 and 1993, respectively. Since 1993, he has been with Toyota Central R\&D Labs., INC. His research interests are advanced control of ac machines and power electronics of hybrid vehicles and electric vehicles. He is a member of the Institute of Electrical Engineers Japan (IEEJ), the Society of Instrument and Control Engineers (SICE), the Society of Automotive Engineers of Japan (JSAE). 